\title{
"No Representation without Taxation" - WSI-Verteilungsbericht 2011
}

\section{Claus Schäfer}

Neue Verteilungsdaten zeichnen das alte Verteilungsbild: Die Arbeitnehmer werden nicht ausreichend am Aufschwung beteiligt, die gesellschaftliche Spaltung wird verfestigt, bei unterentwickelter Binnennachfrage werden Wachstumschancen vergeben. Und über die Griechenland-, Banken- und Euro-Krise droht eine Verschärfung dieser Entwicklung. Fataler Weise versteht die Politik (noch?) nicht, dass Verteilungsungleichheiten und Verteilungsungerechtigkeiten der entscheidende Zusammenhang zwischen diesen nationalen und internationalen Problemlagen sind.

\section{Gesamtwirtschaftliche Entwicklung}

Zur allgemeinen Verteilungsentwicklung ist zunächst Methodisches vorauszuschicken: Das Statistische Bundesamt hat mit seinen im September des Jahres vorgelegten Ergebnissen der Volkswirtschaftlichen Gesamtrechnungen (VGR) zugleich eine völlige Neuberechnung fast aller VGRAggregate bis ins Jahr 1991 zurück präsentiert. Grund ist eine sogenannte "große" Revision, die in den letzten Jahren neu zugängliche Datenquellen ebenso berücksichtigt wie eine EU-weit vereinbarte Neuklassifizierung und -zuordnung von Wirtschaftszweigen und Güterklassen sowie schließlich die Umsetzung seit Jahren „aufgestauter" kleinerer konzeptioneller Korrekturbedarfe (siehe ausführlich: Statistisches Bundesamt 2011a und b). Insgesamt erhöhen die verschiedenen Revisionseffekte das Niveau vieler VGR-Aggregate für den ganzen Revisionszeitraum seit 1991. Aber die Relationen zwischen den Aggregaten haben sich nur wenig geändert. Letztlich ist zwar ein komplett neuer VGR-Datensatz entstanden. „Da aber die Grundmuster der Zeitreihen im Wesentlichen erhalten geblieben sind, dürfte es keine substanziell anderen ökonomischen Bewertungen (der Entwicklung) geben", so das Statistische Bundesamt (2011a, S. 829). Dem kann man sich im Prinzip auch für die Bewertung der Verteilungsentwicklung anschließen.
In den Tabellen 1 bis 3, die dieser Beitrag auf Basis der Berechnungen des Statistischen Bundesamtes präsentiert, wird deshalb auf einen eingehenden Zeitreihenvergleich von Vor- und Nach-Revisionsdaten verzichtet, zumal der beim Statistischen Bundesamt (2011a) detailliert vorgenommen wird. Die abgebildeten Zeitreihen basieren ausschließlich auf den revisionsbedingten Zahlen seit 1991 - und zeigen das "gewohnte“ Bild: Die Lohnquoten gehen brutto und netto langfristig zurück; die Gewinnquoten steigen - jedenfalls bis zum Beginn der Finanzkrise 2008. Der danach einsetzende konjunkturelle Einbruch, der vor allem die Gewinneinkommen nach unten zog, hat diese Langfristentwicklung zwar umgekehrt. Aber auf der Nettoebene ist diese Umkehrung seit 2009 schon weitaus weniger ausgeprägt als auf der Bruttoebene - und klingt auf beiden Ebenen inzwischen auch schon wieder ab. Die Netto-Lohnquote (Spalte A von Tabelle 2) ist im 1. Halbjahr 2011 sogar schon wieder unter das Vorkrisenniveau gesunken, auch wenn das Niveau für das Jahr 2011 insgesamt noch wieder bis auf $43 \%$ steigen könnte. Wegen der entscheidenden Nettoentwicklung ist der verbreitete öffentliche Blick auf die Bruttolohnentwicklung unzureichend. Zwar steigt das Arbeitnehmerentgelt wegen der wieder angezogenen Konjunktur z.B. im 1. Halbjahr 2011 mit 5,1 \% scheinbar stark. Doch 1,4 Prozentpunkte davon sind auf die Zunahme der Beschäftigtenzahl zurückzuführen. Und der gleichzeitig geringe Zuwachs der Unternehmens- und Vermögenseinkommen auf gesamtwirtschaftlicher Ebene um
2,8\% hat mit einem Basiseffekt zu tun, der auf den außerordentlichen Zuwachs dieser Einkommen um 14,5 \% im 1. Halbjahr 2010 zurückgeht (Statistisches Bundesamt 2011c, S. 881). Im Rahmen des verfügbaren Einkommens der privaten Haushalte dagegen sind die Einkommen aus Unternehmertätigkeit und Vermögen vor Steuern immerhin im 1. Halbjahr 2011 um 6,5\% gestiegen (ebd. S. 882).

Es bewahrheitet sich demnach erneut auch am aktuellen Rand, was an Ursachen hinter den Quotenentwicklungen steht: Die Arbeitnehmer bzw. ihre Löhne nehmen nach Abebben der Krise wieder nicht ausreichend am wirtschaftlichen Erfolg teil. Und ob sie vielleicht 2012 eine Chance zum "Nachholen“ haben werden, wie häufig propagiert und erhofft, wird angesichts schon vorhandener und absehbarer Konjunkturrisiken immer unwahrscheinlicher. Statt einer angemessenen Beteiligung am Erfolg werden ihre Lohneinkommen weiter überproportional der Finanzierung des Sozialstaats unterworfen (Tabelle 3). Zwar ist ihre Lohnsteuer-Belastung seit 2009 von hohem Niveau gesunken, aber ihre Beitragsbelastung gleichzeitig gestiegen. Die Gesamtbelastung ist 2010 nur wenig niedriger als 2008 und im 1. Halbjahr 2011

Claus Schäfer, Dr., ist Leiter der Abteilung Wirtschafts- und Sozialwissenschaftliches Institut (WSI) in der Hans-Böckler-Stiftung. Arbeitsschwerpunkt: Verteilungsanalyse von Einkommen und Lebenslagen. e-mail: claus-schaefer@boeckler.de 
sogar schon wieder höher'. Denn zu Beginn 2011 erhöhte sich der Beitragssatz zur gesetzlichen Krankenversicherung von $14,9 \%$ auf 15,5\%, wovon die Arbeitnehmer (unabhängig von Beitragszuschlägen einzelner Kassen) 8,2 \% zu tragen haben, und der zur Arbeitslosenversicherung von $2,8 \%$ auf $3 \%$.

\section{Erwerbseinkommen im Einzelnen}

\subsection{LÖHNE IN UNTERSCHIEDLICHEN ARBEITSVERHÄLTNISSEN}

Die „Beteiligung“ der Arbeitnehmer am (Wieder-)Aufschwung seit 2009/10 wird in der öffentlichen Debatte häufig mit dem zu beobachtenden Zuwachs von Stellen auf dem Arbeitsmarkt und darunter vor allem von sozialversicherungspflichtigen Beschäftigungsverhältnissen gleichgesetzt. Tatsächlich hat sich einerseits die Zunahme von Minijobs zuletzt stark verlangsamt. Aber der seit 2010 bis zum 1. Halbjahr 2011 anhaltende Anstieg auch der sozialversicherungspflichtigen Arbeitsplätze ist nicht per se positiv zu bewerten. Denn laut Statistischem Bundesamt (2011d) tragen neben geringfügiger Beschäftigung die Arbeitsverhältnisse Leiharbeit, Teilzeit und Befristung als sozialversicherungspflichtige Arbeitsverhältnisse rund drei Viertel des gesamten Beschäftigungszuwachses im Jahr 2010. Fortgesetzt wird damit eine Entwicklung seit 2000, die man in Anlehnung an das Deutsche Institut für Wirtschaftsforschung (DIW) als "Jobwunder durch Teilzeit" betiteln kann. Während Teilzeit - ob versicherungspflichtig oder nicht die Entwicklung dominiert, geht Vollzeit im ganzen Zeitraum absolut zurück - und im Ergebnis bleibt das gesamtwirtschaftliche Arbeitsvolumen gleich (Brenke 2011a und b; siehe auch Keller/Seifert 2011). Der Rückgang der Vollzeitstellen seit 2000 wird übrigens durch deren Zuwachs 2010 und danach nicht ausgeglichen.

Teilzeit an sich und prekäre Beschäftigungsverhältnisse in Vollzeit wie Teilzeit, die häufig mit besonders niedrigen Löhnen verbunden sind, erklären also neben der allgemeinen zurückhaltenden Lohnentwicklung einen Großteil des LohnquotenRückgangs. Auch aktuell ist die allgemeine Lohnentwicklung trotz guter Konjunktur

\section{Tabelle 1: Bruttolohn- und -gewinnquote - Deutschland. Das volkswirtschaftliche Einkommen aller Sektoren vor der öffentlichen Umverteilung}

\begin{tabular}{|c|c|c|c|c|c|c|}
\hline & $\begin{array}{c}\text { Volks- } \\
\text { einkommen }\end{array}$ & $\begin{array}{l}\text { Tatsächliche } \\
\text { Brutto- } \\
\text { lohnquote }^{3)}\end{array}$ & $\begin{array}{l}\text { Tatsächliche } \\
\text { Brutto- } \\
\text { gewinnquote }\end{array}$ & Arbeitne & rquote ${ }^{4)}$ & $\begin{array}{c}\text { Struktur- } \\
\text { bereinigte } \\
\text { Bruttolohnquote }^{5)}\end{array}$ \\
\hline & Mrd. $€$ & $\%$ & $\%$ & $\%$ & Index ${ }^{6)}$ & $\%$ \\
\hline & \multicolumn{6}{|c|}{ - alte Bundesländer 1) _ } \\
\hline 1960 & 122,8 & 60,1 & 39,9 & 77,2 & 92,5 & 65,0 \\
\hline 1970 & 274,7 & 67,3 & 32,7 & 83,4 & 100,0 & 67,3 \\
\hline 1980 & 591,0 & 75,2 & 24,8 & 88,3 & 105,9 & 71,0 \\
\hline 1990 & 987,2 & 69,8 & 30,2 & 89,6 & 107,4 & 65,0 \\
\hline \multirow[t]{2}{*}{1991} & 1065,4 & 70,0 & 30,0 & 89,6 & 107,4 & 65,2 \\
\hline & \multicolumn{6}{|c|}{ - alte und neue Bundesländer ${ }^{2)}$ - } \\
\hline 1991 & 1217,2 & 70,8 & 29,2 & 90,8 & 100,0 & 71,0 \\
\hline 1995 & 1423,9 & 71,1 & 28,9 & 89,9 & 96,7 & 73,5 \\
\hline 1996 & 1444,7 & 70,7 & 29,3 & 89,8 & 96,5 & 73,3 \\
\hline 1997 & 1465,7 & 69,9 & 30,1 & 89,6 & 96,3 & 72,6 \\
\hline 1998 & 1492,2 & 70,0 & 30,0 & 89,6 & 97,3 & 71,9 \\
\hline 1999 & 1507,6 & 71,1 & 28,9 & 89,7 & 98,8 & 72,0 \\
\hline 2000 & 1540,9 & 72,1 & 27,9 & 89,9 & 100,7 & 71,6 \\
\hline 2001 & 1577,1 & 71,8 & 28,2 & 89,8 & 100,9 & 71,2 \\
\hline 2002 & 1591,4 & 71,6 & 28,4 & 89,7 & 100,2 & 71,5 \\
\hline 2003 & 1608,5 & 71,0 & 29,0 & 89,4 & 99,0 & 71,7 \\
\hline 2004 & 1686,8 & 67,9 & 32,1 & 89,1 & 98,9 & 68,7 \\
\hline 2005 & 1713,7 & 66,4 & 33,7 & 88,7 & 98,3 & 67,5 \\
\hline 2006 & 1808,7 & 63,9 & 36,1 & 88,6 & 98,8 & 64,7 \\
\hline 20077) & 1877,3 & 63,2 & 36,8 & 88,7 & 100,6 & 62,8 \\
\hline $2008^{7)}$ & 1894,2 & 64,9 & 35,1 & 88,9 & 102,0 & 63,6 \\
\hline 20097) & 1806,2 & 68,2 & 31,8 & 88,9 & 102,1 & 66,8 \\
\hline $2010^{7)}$ & 1897,8 & 66,5 & 33,5 & 88,9 & 102,6 & 64,8 \\
\hline 2011/1 Hj.7) & 945,0 & 66,7 & 33,3 & 88,8 & 103,1 & 64,7 \\
\hline \multicolumn{7}{|c|}{ 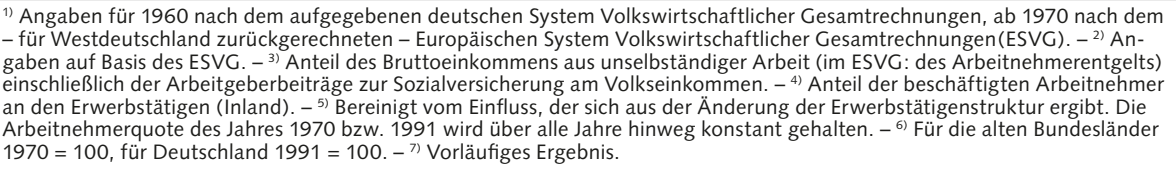 } \\
\hline \multicolumn{7}{|c|}{$\begin{array}{l}\text { Quelle: Statistisches Bundesamt (Fachserie 18: Volkswirtschaftliche Gesamtrechnungen, } \\
\text { Stand: Aug. 2011); Berechnungen des Autors. }\end{array}$} \\
\hline
\end{tabular}

nicht befriedigend. Das zeigen die Lohnveränderungen für drei Hauptkategorien von Beschäftigungsverhältnissen, wie sie hier seit einiger Zeit auf Basis der laufenden Verdiensterhebung des Statistischen Bundesamts (2011e) für die effektiven Arbeitseinkommen - jeweils für West- und Ostdeutschland - abgebildet werden ( $\mathrm{Ta}$ belle 4 und 5).

Demnach sind dort, wo die meisten Personen beschäftigt sind - und prinzipiell den größten Beitrag zur privaten Nachfrage leisten könnten -, nämlich in den Dienstleistungen, die Verdienste 2010 nominal nur verhalten gestiegen. In den westdeutschen Dienstleistungen legten sie z. B. für Vollzeitbeschäftigte auf Stundenbasis ohne (bzw. mit) Sonderzahlungen nur um 1,3\% $(1,1 \%) \mathrm{zu}$, auf Monatsbasis wegen erhöhter Arbeitszeit um 1,7 \% ohne Sonderzahlungen. In den ostdeutschen Dienstleistungen betrug der Anstieg für Vollzeitbeschäftigte auf Stunden- wie auf Monatsbasis bei fast unveränderter Arbeitszeit 1,8 \% bzw. $1,9 \%$ ohne Sonderzahlungen $(1,2 \% \mathrm{mit}$ Sonderzahlungen). Angesichts einer noch gegenzurechnenden Steigerung der Verbraucherpreise um jahresdurchschnittlich 1,1 \% kann dieser Lohnanstieg keinen kräftigen Nachfrageimpuls erzeugen.

Selbst die im Produzierenden Gewerbe 2010 nominal deutlich höheren Lohnzuwächse sind zu relativieren: Von den 4,5\% Anstieg (ohne Sonderzahlungen) bei den Monatsverdiensten entsprechender Vollzeitbeschäftigter in Westdeutschland muss allein mit 1,9 Prozentpunkten ein absoluter Rückgang der Verdienste (ohne Sonderzahlungen) aus 2009 wettgemacht werden. Außerdem gehen die 4,5\% mehr als zur Hälfte auf die 2010 um fast eine

\footnotetext{
1 Auf problematische eingerechnete Sozialversicherungsbeiträge wie darin nicht eingerechnete Gesundheitslasten wurde im letztjährigen Verteilungsbericht hingewiesen (Schäfer 2010).
} 
Tabelle 2: Nettolohn- und -gewinnquote - Deutschland ${ }^{1) 2)}$ Einkommen der privaten Haushalte nach öffentlicher Umverteilung - in \% - 3) 4)

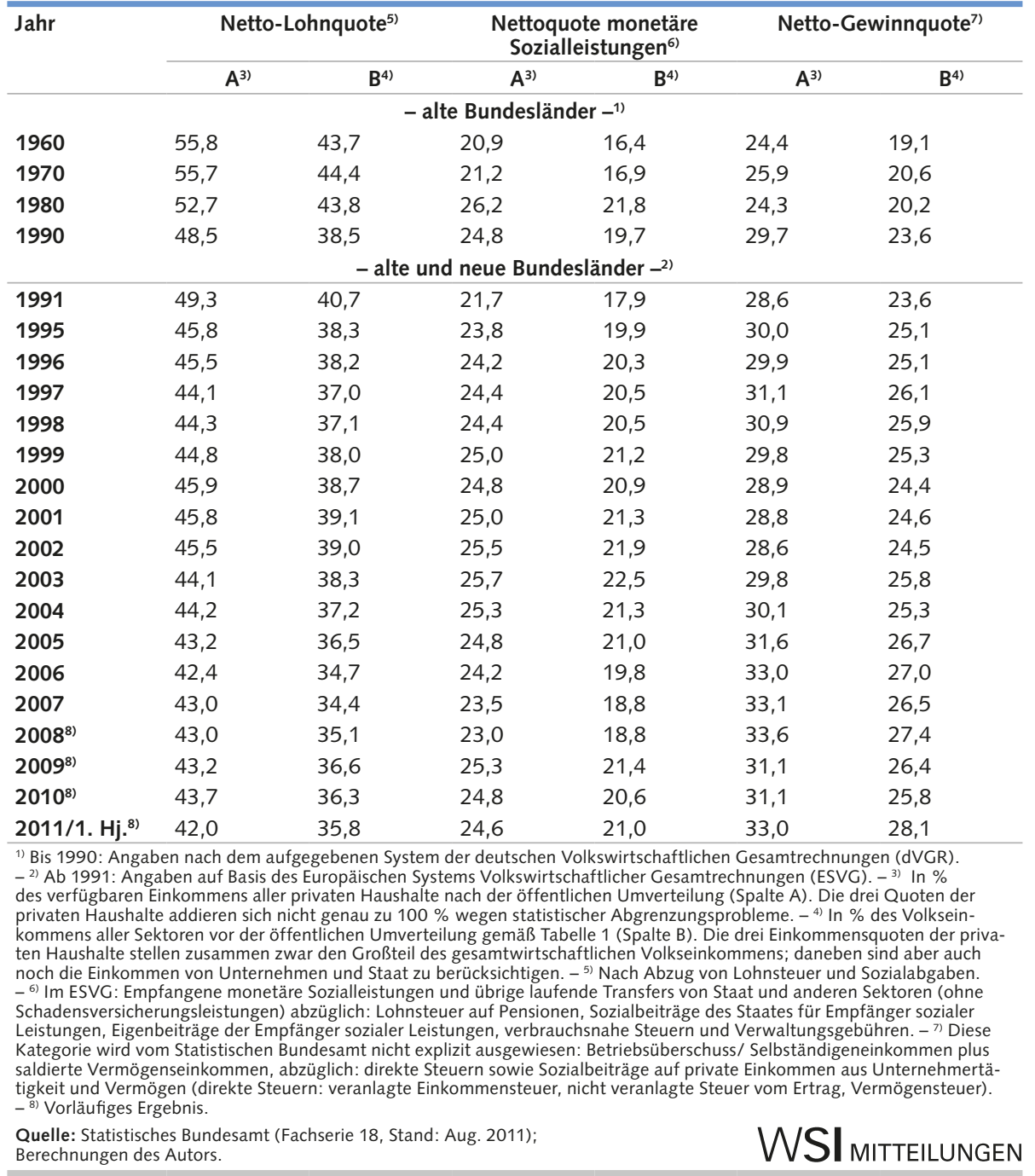

Stunde erhöhte Arbeitszeit zurück. Die Stundenlöhne im westdeutschen Produzierenden Gewerbe sind 2010 bei Vollzeit und ohne Sonderzahlungen nur um 2,1\% gestiegen - genauso stark oder schwach wie im noch krisenbetroffenen Vorjahr. Zwar bessert sich im laufenden Jahr bei den Vollzeitbeschäftigten die Einkommensentwicklung deutlicher: die Bruttomonatsverdienste stiegen zuletzt im 2. Quartal 2011 um 4,2\% bei einem allerdings ebenfalls beschleunigten Preisauftrieb von 2,3\% im Vergleich zum 2. Quartal 2010. Hauptursache sind hier die starken Nominallohnanstiege im Verarbeitenden Gewerbe, bedingt durch eine erneute Zunahme der bezahlten Arbeitszeit sowie gleichgewichtig höhere Stundenlöhne wie höhere Sonderzahlungen (Statistisches Bundesamt 2011f.).
Aber diese kurzfristige Entwicklung in den Jahren 2010/11 wird wieder relativiert, wenn man sie in die längerfristige einfügt, die von überwiegenden Reallohnverlusten gekennzeichnet ist. Entsprechende Berechnungsergebnisse für den Zeitraum 2000 bis 2010 hat im Sommer d.J. das DIW vorgelegt, und zwar mit Hilfe des Sozio-oekonomischen Panels (SOEP) auf Basis von inflationsbereinigten Monatslöhnen (Grabka 2011) wie von preisbereinigten Stundenlöhnen (Brenke/Wagner 2011). ${ }^{2}$ Danach treffen die Reallohnverluste nicht nur den Durchschnitt der individuellen Lohnempfänger (mit ca. $2 \%$ im ganzen Zeitraum), sondern auch - zumindest in den letzten fünf Jahren - alle Gruppen (Dezile) vom untersten bis zum obersten Lohnrand. Die Gruppen mit den niedrigsten Löhnen sind besonders negativ betroffen. Zumindest
Letzteres wird bekräftigt durch eine Studie des Instituts für Arbeitsmarkt- und Berufsforschung (IAB) (Möller 2011), wenn man die dort untersuchten gering qualifizierten Beschäftigten zum Teil mit Niedriglöhnern gleichsetzt. Möller blickt auf Grundlage der „IAB-Stichprobe für integrierte Arbeitsmarktbiographien“ noch weiter zurück bis 1990 und kommt insgesamt zu dem Schluss: „Dabei hat sich die (Reallohn)Schere zwischen niedrigen und mittleren Löhnen in etwa gleich schnell geöffnet wie die zwischen mittleren und höheren Verdiensten“" (ebd., S. 4).

Neben den Lohnzuwächsen sind natürlich auch die erreichten Lohnniveaus von wesentlicher sozialer und ökonomischer Bedeutung, z.B. beim aktuellen Vergleich zwischen ostdeutschen und westdeutschen Niveaus. Die entsprechende Relation in Prozent der westdeutschen Niveaus zeigt mehr oder weniger unveränderte Lohnlücken im Osten (viertletzte und letzte Spalte in Tabelle 5). Dort ist der Vergleich auf Stundenbasis vorgenommen worden und ergibt für das Produzierende Gewerbe und darin Vollzeit (Teilzeit) ein Anpassungsniveau an den Westen von lediglich $66,8 \%(63,7 \%)$ ohne Sonderzahlungen - und von $64,3 \%(61,0 \%)$ mit Sonderzahlungen. Mit durchschnittlich weniger Sonderzahlungen und gleichzeitig mehr Arbeitszeit (rund 1,1 Wochenstunden) als im Westen werden die ostdeutschen Beschäftigten zusätzlich „diskriminiert“. Da ist es wenig Trost, dass durch die längere Arbeitszeit in den neuen Bundesländern der Verdienstabstand zum Westen auf Monatsbasis etwas geringer ausfällt. Wenn der Fachkräftemangel und sogar der Arbeitskräftemangel für Ostdeutschland besonders beklagt wird, dann hat er in der Lohnlücke, die seit 15 Jahren besteht, eine wesentliche Ursache, die bis heute junge und qualifizierte Erwerbsfähige ,auswandern" und nicht zurückkehren lässt (siehe z. B. Der Tagesspiegel 2011).

\subsection{GEWINNE DER PRODUKTIONS- UNTERNEHMEN}

Angesichts der 2008 eingesetzten weltweiten Finanz- und Wirtschaftskrise gab es für die zukünftige Entwicklung der Gewinneinkommen deutscher Unternehmen besonders negative Befürchtungen, weil

2 Der Autor dankt Markus Grabka/DIW für die zur Verfügung gestellten Berechnungen. 
zunächst Umsätze und Erlöse vor allem in den exportorientierten Produktionsbereichen stark einbrachen. Aber schon im Verlauf des Jahres 2009 verbesserten sich bekanntlich die Rahmenbedingungen wieder, sodass sich die ursprünglichen Sorgen insgesamt - bei großen Unterschieden zwischen einzelnen Branchen - nicht bewahrheiteten. Zwar hat die Deutsche Bundesbank ihre traditionelle detaillierte Berichterstattung über „Ertragslage und Finanzierungsverhältnisse deutscher Unternehmen" für 2010 und erst recht auch für das laufende Jahr bisher nicht vorgelegt. Aber die für 2009 veröffentlichte entsprechende Analyse (Deutsche Bundesbank 2010) zeichnet selbst für dieses Krisenjahr ein erstaunlich robustes Bild der Ertragslage und verfügbaren Finanzmittel. Aus Sicht der Bundesbank waren dafür - neben der expansiven staatlichen Wirtschafts- und Finanzpolitik inklusive geförderter Kurzarbeit und einer verhaltenen Tariflohnentwicklung einschließlich genutzter Öffnungsklauseln - vor allem die in den Vorjahren gewonnenen Polster der Unternehmen durch überdurchschnittliche Gewinnentwicklung verantwortlich.

Die Bundesbank schreibt z.B.: „Die Gesamtzahl der Unternehmenspleiten erreichte im Berichtszeitraum (2009) bei Weitem nicht den Stand der vorherigen Abschwungphase 2002/2003“. Und weiter: „Trotz des Umsatz- und Erlöseinbruchs erreichte das „Bruttojahresergebnis... im Krisenjahr gleichwohl fast das Niveau von 2005 und war damit deutlich höher als in der recht milden konjunkturellen Schwächephase Anfang der letzten Dekade. Insofern ist die Ertragslage der Unternehmen im Berichtszeitraum noch als vergleichsweise günstig einzustufen. Diese Einordnung bestätigt sich auch mit Blick auf die Brutto-Umsatzrendite, die sich 2009 um einen viertel Prozentpunkt auf 4 1/4 \% ermäßigte. Der Höchststand aus dem Boomjahr 2007 wurde damit um einen Prozentpunkt verfehlt. Dennoch lag die so gemessene Rentabilität der Betriebe weiter spürbar über den Werten der Jahre 2002 und 2003. Die Nettoumsatzrendite, die sich nach Abzug der Ertragsteuern vom Jahresergebnis entsprechend berechnet, sank im Berichtszeitraum auf $31 / 4 \%$, nach $3 \frac{1}{2} \%$ im Jahr 2008 und $4 \%$ im Jahr 2007. Insgesamt konnte die Vielzahl der Unternehmen in den hier erfassten Wirtschaftsbereichen selbst unter den sehr schwierigen Bedingungen der Finanz- und

Tabelle 3: Abgabenbelastung von Arbeits- und Kapitaleinkommen der privaten Haushalte - Deutschland - in \%-1)

\begin{tabular}{|c|c|c|c|c|}
\hline Jahr & $\begin{array}{l}\text { Lohnsteuer- } \\
\text { belastung der } \\
\text { Bruttolöhne } \\
\text { und -gehälter }\end{array}$ & $\begin{array}{l}\text { Belastungen durch } \\
\text { Sozialbeiträge auf } \\
\text { Bruttolöhne und } \\
\text { gehälter }\end{array}$ & $\begin{array}{l}\text { Belastung durch } \\
\text { direkte Steuern's) auf } \\
\text { Gewinn- und } \\
\text { Vermögens- } \\
\text { einkommen }\end{array}$ & $\begin{array}{l}\text { Belastung durch } \\
\text { Sozialbeiträge } \\
\text { auf Gewinn- und } \\
\text { Vermögens- } \\
\text { einkommen }\end{array}$ \\
\hline & \multicolumn{4}{|c|}{ - alte Bundesländer - } \\
\hline 1960 & 6,3 & 9,4 & 20,0 & 3,0 \\
\hline 1970 & 11,8 & 10,7 & 16,1 & 2,9 \\
\hline 1980 & 15,8 & 12,8 & 15,3 & 3,9 \\
\hline \multirow[t]{2}{*}{1990} & 16,2 & 14,2 & 9,8 & 3,0 \\
\hline & \multicolumn{4}{|c|}{ - alte und neue Bundesländer - } \\
\hline 1991 & 15,9 & 14,0 & 8,6 & 3,1 \\
\hline 1995 & 18,3 & 15,3 & 5,3 & 3,7 \\
\hline 1996 & 17,8 & 15,7 & 4,2 & 4,1 \\
\hline 1997 & 17,9 & 16,4 & 3,4 & 3,8 \\
\hline 1998 & 17,9 & 16,4 & 4,6 & 3,7 \\
\hline 1999 & 17,9 & 16,1 & 6,2 & 3,7 \\
\hline 2000 & 17,4 & 15,9 & 7,8 & 3,7 \\
\hline 2001 & 16,7 & 15,8 & 7,9 & 3,7 \\
\hline 2002 & 16,7 & 15,9 & 6,8 & 3,7 \\
\hline 2003 & 16,8 & 16,3 & 5,5 & 3,4 \\
\hline 2004 & 15,8 & 16,3 & 5,3 & 3,4 \\
\hline 2005 & 15,5 & 16,6 & 5,8 & 3,1 \\
\hline 2006 & 15,8 & 17,0 & 7,0 & 2,9 \\
\hline $2007^{4)}$ & 16,3 & 16,8 & 8,2 & 3,0 \\
\hline 20084) & 16,7 & 17,0 & 8,6 & 2,9 \\
\hline 20094) & 16,2 & 17,6 & 8,8 & 4,2 \\
\hline 20104) & 15,3 & 17,7 & 8,2 & 3,9 \\
\hline 2011/1. $\mathrm{Hj}^{4)}$ & 15,8 & 18,2 & 8,6 & 3,6 \\
\hline \multicolumn{5}{|c|}{$\begin{array}{l}\text { 1) In \% der jeweiligen Bruttoeinkommen. - 2) Veranlagte Einkommensteuer; nicht veranlagte Steuer vom Ertrag; Vermögen- } \\
\text { steuer. - }{ }^{3)} \text { Pflicht- und freiwillige Beiträge von Selbständigen (und Nichtselbständigen). - }{ }^{4} \text { Vorläufiges Ergebnis. }\end{array}$} \\
\hline
\end{tabular}

Wirtschaftskrise eine Rendite erwirtschaften, die ausreichend war, um das Vertrauen der Eigen- und Fremdkapitalgeber in die Tragfähigkeit der Geschäftsmodelle zu erhalten" (Deutsche Bundesbank 2010, S. 34). „Die Eigenmittelausstattung aller erfassten Unternehmen ging 2009 leicht zurück (-1 1 1/2\%), nahm im Verhältnis zur Bilanzsumme jedoch weiter zu. Die Eigenmittelquote lag etwas über $25 \%$ und damit etwa einen halben Prozentpunkt über den Ergebnissen der beiden Vorjahre" (ebd. S. 43f.). „Die Stärkung der Risikotragfähigkeit, die sich an der Eigenkapitalunterlegung der Aktiva bemisst, hat sich 2009 mit Blick auf den Unternehmenssektor als Ganzes fortgesetzt“ (ebd. S. 44).

Zur voraussichtlichen Entwicklung 2010 vermerkt schließlich die Bundesbank in ihrem Bericht, der Ende 2010 mit schon einiger Kenntnis der entsprechenden Entwicklung publiziert wurde: „Auswertungen von Abschlüssen größerer börsennotierter Konzerne für die ersten neun Monate im Jahr 2010 sind ein Hinweis darauf, dass sich die Brutto-Umsatzrendite inzwischen dem Vorkrisenniveau wieder angenähert haben könnte“ (S. 44). Diese Vorhersage hat die Bundesbank (Deutsche Bundesbank 2011) kürzlich erst für den ganzen Unternehmenssektor in einem mager kommentierten Zahlenwerk über die „Ergebnisse der Gesamtwirtschaftlichen Finanzierungsrechnung für Deutschland 2005-2010“ weitgehend bestätigt. Vor allem aber hat sie darin mit vielen Kennziffern noch einmal die außerordentlich gute Gewinn- und Eigenkapitalentwicklung über einen längeren Zeitraum einschließlich der krisenbelasteten Jahre 2008 und 2009 eindrucksvoll betont, in der auch die Dominanz betrieblicher Geldvermögensbildung im weiteren Sinn gegenüber der Sachvermögensbildung hervorsticht. Das gilt auch für 2009 und selbst wieder für 2010 (siehe im Einzelnen ebd., S. 68): Im letzten Jahr haben die Produktionsunternehmen (in der Bundesbank-Begrifflichkeit die „Nicht-finanziellen Kapitalgesellschaften“) zwar Netto-Investitionen nach Abschreibungen in Höhe von $62 \mathrm{Mrd}$. € getätigt, aber sie haben für 94 Mrd. Geldvermögen im engeren Sinn gebildet. Darunter haben sie für $37 \mathrm{Mrd}$. fremde Aktien 
Tabelle 4: Durchschnittliche effektive Arbeitseinkommen in Westdeutschland 2010 (brutto) Ergebnisse der Verdienststatistik ${ }^{1)}$ für verschiedene Beschäftigungsverhältnisse

\begin{tabular}{|c|c|c|c|c|c|c|c|c|c|c|c|}
\hline \multirow{3}{*}{\multicolumn{2}{|c|}{$\begin{array}{l}\text { Wirtschaftsbereich/ } \\
\text { Beschäftigungsverhältnis }\end{array}$}} & \multirow{2}{*}{\multicolumn{2}{|c|}{$\begin{array}{l}\text { Bezahlte Wochen- } \\
\text { arbeits-Stunden }\end{array}$}} & \multirow{2}{*}{\multicolumn{2}{|c|}{$\begin{array}{l}\text { Monatsverdienst ohne } \\
\text { Sonderz. }\end{array}$}} & \multirow{3}{*}{$\begin{array}{c}\text { Monatliche } \\
\text { Sonderzahlung } \\
€\end{array}$} & \multicolumn{5}{|c|}{ Stundenverdienst } \\
\hline & & & & & & & \multicolumn{3}{|c|}{ ohne Sonderz. } & \multicolumn{2}{|c|}{ mit Sonderz. } \\
\hline & & Std. & $\%^{2)}$ & $€$ & $\%^{2)}$ & & $\%^{2)}$ & $€$ & $\%^{2)}$ & $€$ & $\%^{2)}$ \\
\hline \multicolumn{12}{|c|}{ Produzierendes Gewerbe } \\
\hline \multirow[t]{3}{*}{ Vollzeit } & alle & 37,8 & 2,4 & 3395 & 4,5 & 396 & 3,0 & 20,69 & 2,1 & 23,10 & 1,9 \\
\hline & Männer & 37,8 & 2,5 & 3515 & 4,5 & 412 & 2,4 & 21,38 & 2,1 & 23,88 & 1,8 \\
\hline & Frauen & 37,3 & 2,1 & 2785 & 4,4 & 314 & 6,7 & 17,17 & 2,3 & 19,10 & 2,5 \\
\hline \multirow[t]{3}{*}{ Teilzeit } & alle & 23,8 & 2,0 & 1695 & 3,6 & 177 & 6,0 & 16,39 & 1,6 & 18,10 & 1,8 \\
\hline & Männer & 25,2 & 2,3 & 2139 & 4,1 & 263 & 9,7 & 19,51 & 1,7 & 21,91 & 2,3 \\
\hline & Frauen & 23,6 & 1,9 & 1622 & 3,4 & 163 & 4,9 & 15,84 & 1,5 & 17,43 & 1,6 \\
\hline \multirow[t]{3}{*}{ Minijob ${ }^{3)}$} & alle & -- & -- & 303 & 0,3 & -- & -- & -- & -- & -- & -- \\
\hline & Männer & -- & -- & 303 & 2,1 & -- & -- & -- & -- & -- & -- \\
\hline & Frauen & -- & -- & 303 & $-0,7$ & -- & -- & -- & -- & -- & -- \\
\hline \multicolumn{12}{|c|}{ Dienstleistungen } \\
\hline \multirow[t]{3}{*}{ Vollzeit } & alle & 39,4 & 0,3 & 3302 & 1,7 & 307 & $-0,6$ & 19,30 & 1,3 & 21,09 & 1,1 \\
\hline & Männer & 39,6 & 0,4 & 3556 & 1,6 & 351 & $-0,5$ & 20,64 & 1,2 & 22,68 & 1,0 \\
\hline & Frauen & 38,9 & 0,3 & 2875 & 1,7 & 233 & $-1,0$ & 16,99 & 1,5 & 18,37 & 1,3 \\
\hline \multirow[t]{3}{*}{ Teilzeit } & alle & 23,9 & 0,6 & 1641 & 2,1 & 114 & $-0,9$ & 15,79 & 1,5 & 16,89 & 1,3 \\
\hline & Männer & 24,9 & 0,6 & 1729 & 2,1 & 116 & $-4,4$ & 15,99 & 1,5 & 16,97 & 1,1 \\
\hline & Frauen & 23,8 & 0,6 & 1627 & 2,1 & 115 & $-0,4$ & 15,76 & 1,5 & 16,87 & 1,3 \\
\hline \multirow[t]{3}{*}{ Minijob $^{3)}$} & alle & -- & -- & 276 & 2,5 & -- & -- & -- & -- & -- & -- \\
\hline & Männer & -- & -- & 265 & 3,8 & -- & -- & -- & -- & -- & -- \\
\hline & Frauen & -- & -- & 282 & 1,9 & -- & -- & -- & -- & -- & -- \\
\hline \multicolumn{12}{|c|}{ Insgesamt } \\
\hline \multirow{2}{*}{\multicolumn{2}{|c|}{ Westdeutschland ${ }^{4)}$}} & 35,4 & 0,9 & 2958 & 2,5 & 292 & 0,4 & 19,22 & 1,5 & 21,12 & 1,4 \\
\hline & Deutschland ${ }^{4)}$ & 35,6 & 0,8 & 2871 & 2,4 & 271 & 0,1 & 18,54 & 1,6 & 20,30 & 1,4 \\
\hline
\end{tabular}

1) Arbeitnehmerverdienste“ in der Fachserie 16, Reihe 2.3 des Statistischen Bundesamts; Westdeutschland inkl. Berlin . ${ }^{2)}$ Veränderung gegenüber dem Vorjahr. - ${ }^{3)}$ Für Minijobber enthält die Statistik keine Angaben zu Arbeitszeit und zu Sonderzahlungen. Minijobber erhalten per se keine Sonderzahlungen (oder sie werden auf den Verdienst angerechnet.) - ${ }^{4)}$ Ohne Minijobber.

Quelle: Statistisches Bundesamt 2011 (Verdienststatistik); Berechnungen des Autors.

WSI MITTEILUNGEN

Tabelle 5: Durchschnittliche effektive Arbeitseinkommen in Ostdeutschland 2010 (brutto) Ergebnisse der Verdienststatistik ${ }^{1)}$ für verschiedene Beschäftigungsverhältnisse

\begin{tabular}{|c|c|c|c|c|c|c|c|c|c|c|c|c|c|}
\hline \multirow{3}{*}{\multicolumn{2}{|c|}{$\begin{array}{l}\text { Wirtschaftsbereich/ } \\
\text { Beschäftigungsverhältnis }\end{array}$}} & \multirow{2}{*}{\multicolumn{2}{|c|}{$\begin{array}{l}\text { Bezahlte Wochen- } \\
\text { arbeits-Stunden }\end{array}$}} & \multirow{2}{*}{\multicolumn{2}{|c|}{$\begin{array}{l}\text { Monatsverdienst } \\
\text { ohne Sonderz. }\end{array}$}} & \multirow{2}{*}{\multicolumn{2}{|c|}{$\begin{array}{l}\text { Monatliche } \\
\text { Sonderzahlung }\end{array}$}} & \multicolumn{6}{|c|}{ Stundenverdienst } \\
\hline & & & & & & & & \multicolumn{3}{|c|}{ ohne Sonderz. } & \multicolumn{3}{|c|}{ mit Sonderz. } \\
\hline & & Std. & $\%^{2)}$ & $€$ & $\%^{2)}$ & $€$ & $\%^{2)}$ & $€$ & $\%^{2)}$ & $\%^{5)}$ & $€$ & $\%^{2)}$ & $\%^{5)}$ \\
\hline \multicolumn{14}{|c|}{ Produzierendes Gewerbe } \\
\hline \multirow[t]{3}{*}{ Vollzeit } & alle & 38,9 & 1,5 & 2335 & 3,2 & 177 & 6,7 & 13,82 & 1,7 & 66,8 & 14,86 & 1,9 & 64,3 \\
\hline & Männer & 38,9 & 1,5 & 2423 & 3,2 & 184 & 5,9 & 14,33 & 1,6 & 67,0 & 15,42 & 1,8 & 64,6 \\
\hline & Frauen & 38,9 & 1,5 & 2010 & 3,4 & 154 & 10,4 & 11,90 & 1,9 & 69,3 & 12,81 & 2,4 & 67,1 \\
\hline \multirow[t]{3}{*}{ Teilzeit } & alle & 29,0 & 1,8 & 1317 & 4,9 & 75 & 15,9 & 10,44 & 3,0 & 63,7 & 11,04 & 3,6 & 61,0 \\
\hline & Männer & 29,0 & 4,3 & $1634^{6)}$ & $--6)$ & 144 & 34,4 & 14,53 & 7,3 & 74,5 & 15,67 & 8,6 & 71,5 \\
\hline & Frauen & 29,0 & 1,2 & 1211 & 3,4 & 61 & 9,9 & 9,60 & 2,1 & 60,6 & 10,08 & 2,4 & 57,8 \\
\hline \multirow[t]{3}{*}{ Minijob $^{3)}$} & alle & -- & -- & 261 & 9,4 & -- & -- & -- & -- & -- & -- & -- & -- \\
\hline & Männer & -- & -- & 267 & 5,1 & -- & -- & -- & -- & -- & -- & -- & -- \\
\hline & Frauen & -- & -- & 256 & 13,0 & -- & -- & -- & -- & -- & -- & -- & -- \\
\hline \multicolumn{14}{|c|}{ Dienstleistungen } \\
\hline \multirow[t]{3}{*}{ Vollzeit } & alle & 39,5 & 0,1 & 2679 & 1,9 & 156 & $-7,9$ & 15,59 & 1,8 & 80,8 & 16,50 & 1,2 & 78,2 \\
\hline & Männer & 39,9 & 0,2 & 2734 & 1,9 & 159 & $-8,5$ & 15,77 & 1,8 & 76,4 & 16,69 & 1,1 & 73,6 \\
\hline & Frauen & 39,2 & 0,1 & 2616 & 1,8 & 153 & $-7,2$ & 15,38 & 1,8 & 90,5 & 16,27 & 1,2 & 88,6 \\
\hline \multirow[t]{3}{*}{ Teilzeit } & alle & 28,8 & 0,0 & 1679 & 1,8 & 92 & $-4,7$ & 13,41 & 1,8 & 84,9 & 14,15 & 1,4 & 83,8 \\
\hline & Männer & 28,8 & $-0,4$ & 1661 & 0,7 & 81 & $-9,9$ & 13,29 & 1,1 & 83,1 & 13,94 & 0,6 & 82,1 \\
\hline & Frauen & 28,8 & 0,1 & 1682 & 1,9 & 94 & $-4,0$ & 13,43 & 1,8 & 85,2 & 14,18 & 1,5 & 84,1 \\
\hline \multirow[t]{3}{*}{ Minijob $^{3)}$} & alle & -- & -- & 204 & 4,6 & -- & -- & -- & -- & -- & -- & -- & -- \\
\hline & Männer & -- & -- & 200 & 6,2 & -- & -- & -- & -- & -- & -- & -- & -- \\
\hline & Frauen & -- & -- & 206 & 3,6 & -- & -- & -- & -- & -- & -- & -- & -- \\
\hline \multicolumn{14}{|c|}{ Insgesamt } \\
\hline \multicolumn{2}{|c|}{ Ostdeutschland ${ }^{4)}$} & 36,9 & 0,4 & 2342 & 2,2 & 147 & $-2,8$ & 14,61 & 1,7 & 76,0 & 15,53 & 1,4 & 73,5 \\
\hline \multicolumn{2}{|c|}{ Deutschland4) } & 35,6 & 0,8 & 2871 & 2,4 & 271 & 0,1 & 18,54 & 1,6 & -- & 20,30 & 1,4 & -- \\
\hline \multicolumn{14}{|c|}{$\begin{array}{l}\text { 1) Arbeitnehmerverdienste “ in der Fachserie 16, Reihe } 2.1 \text { des Statistischen Bundesamts: Verdienste und Arbeitskosten; Ostdeutschland ohne Berlin. - }{ }^{2)} \text { Veränderung gegenüber dem Vorjahr. - } \\
\text { 3) "Für Minijobber enthält die Statistik keine Angaben zu Arbeitszeit und zu Sonderzahlungen. Minijobber erhalten per se keine Sonderzahlungen (oder sie werden auf den Verdienst angerechnet.) } \\
\text { - (4) Ohne Minijobber. - }{ }^{5)} \text { Relation des ostdeutschen Verdienstniveaus zum jeweiligen westdeutschen Niveau in \%. - 6) Wegen zu geringer Fallzahlen werden vom Statistischen Bundesamt für } \\
2010 \text { keine Monatsverdienste angegeben. Deshalb stehen hier noch die Angaben für } 2009 \text { - die in } 2010 \text { um ca. } 5 \% \text { höher ausfallen dürften. }\end{array}$} \\
\hline
\end{tabular}


gekauft, für 39 Mrd. sonstige Anteilsrechte erworben und mit $27 \mathrm{Mrd}$. € für „Finanzderivate" bezahlt. Und sie haben schließlich durch Formen der Außenfinanzierung weiteren finanziellen Spielraum erzielt, im Wesentlichen durch Ausgabe bzw. Verkauf eigener Wertpapiere.

\section{Personelle Verteilung}

\subsection{FORTGESETZTE POLARISIERUNG}

Auch mit neueren Informationen zur personellen Verteilung lässt sich das bekannte Bild zunehmender Ungleichheit fortmalen. Einerseits geben Schätzungen auf Basis plausibler Wirtschafts- und Bankeninformationen einen Zuwachs von Zahl und Vermögensbestand der „Superreichen" schon kurz nach der letzten Finanzmarktkrise auch für Deutschland bekannt (vgl. z.B. Manager-Magazin 2011; Merrill Lynch/Capgemini 2011). Aber auch unterhalb dieser absoluten „Spitze“ nehmen die Einkommen z.B. von Unternehmensvorständen weiter teils kräftig zu, wie diverse belastbarere Untersuchungen zeigen. So geht z.B. aus einer repräsentativen Befragung im Auftrag des Handelsblatts bei GmbH-Geschäftsführern hervor, dass deren Gehälter 2010 gegenüber dem Vorjahr um 8,1 \% gestiegen sind, gemessen am Median-Wert (118.473€), und sogar um $10,1 \%$, wenn die Steigerung am arithmetischen Mittel (146.980€) abgelesen wird (Handelsblatt vom 21.10.11, S. 29). Zu diesem Festgehalt kommen Tantiemen, Pensionszusagen und weitere betriebliche Leistungen hinzu. Solche Personen und ihre Wohlfahrtslage lassen sich bekanntlich kaum mit amtlicher Statistik oder Haushaltsbefragungen der Wissenschaft erfassen - die schon deshalb das Ausmaß von Reichtum und damit auch von Ungleichheit immer unterschätzen müssen. ${ }^{3}$

Andererseits weisen die aktuellen Ergebnisse von wissenschaftlichen Haushaltsbefragungen, auf die Politik und Öffentlichkeit nach wie vor zur Darstellung der Einkommens- und Vermögensverteilung angewiesen sind, einen weiteren Zuwachs an Armut aus, zumindest eine weitere Verfestigung von Armut - obwohl die Wirtschaftskrise schon im Verlauf des Jahres 2009 durch ein Wiederanziehen der Konjunktur überwunden schien. So setzt sich auf Basis von Daten des Mikrozensus, die die Bundesregierung (2011) im Sommer dieses Jahres zur Beantwortung einer parlamentarischen Anfrage verwendet hat, die leichte Aufwärtsbewegung der Armutsquote nach EU-Definition fort, die seit einiger Zeit verbrämend Armutsgefährdungsquote genannt wird: von 14,0 \% der Gesamtbevölkerung (2006) mit jährlich kleinen Schritten kontinuierlich bis auf 14,6 \% (2009) (ebd., S. 3). ${ }^{4}$ Auch auf Grundlage der EU-weiten Erhebung EU-SILC, die seit Kurzem für Deutschland mit dem SOEP identisch ist, lässt sich von 2007 auf 2008 ein Anstieg der deutschen Armutsquote in EU-Abgrenzung von $15,2 \%$ auf $15,5 \%$ feststellen. Das ist dem „Datenreport 2011“ zu entnehmen, der alle zwei Jahre von Statistischem Bundesamt, Wissenschaftszentrum Berlin (WZB) und Bundeszentrale für politische Bildung (BpB) erarbeitet und veröffentlicht wird (Statistisches Bundesamt et al. 2011, S. 154). An anderer Stelle im Datenreport 2011 wird die EU-definierte Armutsquote für die deutsche Bevölkerung 2009 mit „nur“ $12,3 \%$ angegeben. Doch für das Jahr 2009 standen für den Report aus dem SOEP nur Befragten-Angaben über laufende Monatseinkommen zur Verfügung, die immer niedriger und unvollständiger sind als die vorerst bis 2008 verfügbaren Angaben zu Jahreseinkommen einschließlich von Sonderzahlungen, Boni, Prämien, Gewinnanteilen, Zinsen usw.

Viel entscheidender sind aber die in im Datenreport enthaltenen Informationen über die vollzogene Spreizung und Verfestigung der gesamten Einkommensverteilung, die an mehreren Indikatoren im Längsschnittvergleich bis 1993 und sogar teilweise bis 1985 zurück festgemacht werden: Auf Grundlage der SOEP-Erhebungen zum monatlichen Haushaltsnettoeinkommen haben sich „Alle Indizes... gegenüber 2001 vergrößert, das Ausmaß an Niedrigeinkommen und Armut ist auf eines der höchsten Niveaus der letzten beiden Jahrzehnte angestiegen. Zugleich haben sich die Einkommen der Armen immer weiter von der Armutsschwelle entfernt, und die Intensität der Armut hat sich erhöht. In den letzten Jahren setzt sich dieser Trend indes nicht weiter fort, Armuts- und Ungleichheitsziffern stagnieren derzeit auf allerdings höherem Niveau als noch in den beiden Dekaden zuvor. Auch bei der jahresbezogenen Einkommensberechnung weisen alle Indikatoren auf eine langjäh- rige Zunahme der Betroffenheit und eine wachsende Intensität der Einkommensarmut hin; aber auch bei diesem Einkommenskonzept zeigt sich in den letzten Jahren eine Stagnation im Armuts- und Ungleichheitsverlauf“ (ebd. S. 165).

In diesem Gesamtrahmen zeigt sich bei einigen Indikatoren dennoch eine aktuelle Fortsetzung von Polarisierung, wenn die Verweildauern und Betroffenheitshäufigkeiten im Armuts- und im Reichtums"sektor" analysiert werden. Das geschieht im Datenreport 2011 mit Hilfe einer Aufteilung der privaten Haushalte in Quintile, d.h. in insgesamt fünf gleich große Bevölkerungsgruppen von je $20 \%$, geordnet nach ihren aufsteigenden Einkommen. Danach hat sich das Risiko, innerhalb von vier Jahren im untersten Quintil mit den niedrigsten Einkommen zu verbleiben, über die Jahre hinweg deutlich erhöht: „Von 57 \% in den 1980er Jahren auf $65 \%$ in 2009. Der Übergang von der untersten in gehobene Einkommenslagen hat sich entsprechend verringert" (ebd., S. 171). Gleichzeitig ist der Verbleib im obersten Quintil mit den höchsten Einkommen zwischenzeitlich nur wenig verändert: Sind dort in den 1980er und 90er Jahren gut 66 \% der Einkommensbezieher innerhalb von vier Jahren in diesem Einkommensquintil verblieben, waren es nach einem vorübergehend leichten Anstieg um die Jahrtausendwende zuletzt 2006 bis 2009 wieder knapp 66 \% (ebd.). Die Ergebnisse dieser „Mobilitätsanalyse“ von Einkommen zeigen auch, „dass fast 87 \% der Personen, die im Jahr 2009 in der untersten Einkommensschicht und damit in relativer Einkommensarmut lebten, bereits in den vier Vorjahren (2005 bis 2008) zumindest einmal von Armut betroffen waren, darunter sind gut ein Drittel der Personen in diesem Zeitraum dauerhaft arm“ (ebd., S. 172). Für diese unterste Einkommensschicht gilt auch: „Der Anteil an Personen, die im zurückliegenden Zeitraum von vier Jahren mindestens ein Mal unter der Armutsgrenze lagen, hat zugenommen, wobei insbesondere mehr-

3 Gäbe es allerdings z.B. eine Vermögensteuer in Deutschland, ließen sich zumindest die Vermögen auch dieser Personen relativ gut bestimmen und damit indirekt, über Vermögensveränderungen, auch deren tendenzielle Einkommensentwicklung.

4 Die Armuts(gefährdungs)quote umfasst alle Netto-Äquivalenzeinkommen, die unter der Armutsschwelle von $60 \%$ des Einkommensmedians aller Haushaltseinkommen in Deutschland liegen. 
fache oder dauerhafte Armutserfahrungen angestiegen sind“ (ebd.).

Weitere Indikatoren auf SOEP-Basis im Datenreport 2011 bestätigen noch einmal für die Haushaltsebene, was in Abschnitt 1 dieses Beitrags zur gesamtwirtschaftlichen Verteilungsentwicklung bereits ausgeführt wurde: Die durchschnittliche Abgabenlast der Arbeitnehmer ist fast kontinuierlich seit den 90er Jahren gestiegen (ebd., S. 135); die Haushalts-Nettoeinkommen in Westdeutschland haben seit 2003 um nur 3,3\% zugelegt, in Ostdeutschland sind sie konstant geblieben (ebd., S. 137); das Nettoeinkommen der ostdeutschen Haushalte hat sich auch deshalb seit 2003, als es „schon“ $78 \%$ des durchschnittlichen westdeutschen Nettoeinkommens ausmachte, bis 2008 wieder auf $75 \%$ des entsprechenden Niveaus im Westen vermindert (ebd., S.136).

\section{2 ÖFFENTLICHE UMVERTEILUNG}

Armut und Reichtum bei verfügbaren Einkommen sind auch wesentlich Ergebnis von vorangegangener Umverteilung des Staates - nicht nur von Marktprozessen. Und seit Langem ist bekannt, dass insbesondere diverse Steuerentlastungen seit den 1990er Jahren zugunsten von hohen Einkommen und Vermögen eine zunehmende Spreizung der letztlich ausgabefähigen Einkommen gefördert haben - begonnen mit der Aussetzung der Vermögensteuer unter Helmut Kohl, mit einem "Höhepunkt“ gemessen an quantitativen Dimensionen durch die Einkommensteuerreformen unter Gerhard Schröder und einer Fortsetzung durch die zuletzt eingeführte Abgeltungssteuer auf Zins- und Veräußerungserträge unter Angela Merkel. Nun wird immer wieder der Versuch gemacht, trotz dieser einseitigen Entlastungen den gesamten Umverteilungsmechanismus des Staates und speziell auch des Abgabensystems als intakt bzw. gerecht darzustellen. Doch das ist allein schon wegen „einfacher" gesamtwirtschaftlicher Daten fragwürdig, wie z.B. Tabelle 3 zeigt, nach der zumindest die Einkommensquellen Gewinn und Vermögen sehr viel weniger als die Lohnquelle zur Finanzierung des Sozialstaates herangezogen werden.

Solche Beschwichtigungsversuche werden nun gerade mit Hilfe vermeintlich genauerer personeller Lastenverteilungen unternommen. So beruft sich z. B. das Handelsblatt (vom 17.10.2011, S. 17) auf eine exklusive Untersuchung zur Steuerbelastung von Managern im internationalen Vergleich „unter vereinfachenden Annahmen", die für deutsche Spitzenverdiener eine durchschnittliche Einkommensteuerlast von $40 \%$ ermittelt haben will. Und auch das Statistische Bundesamt (2011f) bedient - wenn auch unfreiwillig - mit einer Pressemeldung zu ersten Ergebnissen der Einkommensteuerstatistik 2007 als letztverfügbarem Berichtsjahr die Behauptung von einer „korrekten“ Lastenverteilung - wobei bei mancher Ergebnisrezeption in den Medien aus der Lohn- bzw. Einkommensteuer sogar „alle Steuern“ gemacht werden. Laut dieser Pressemeldung zahlen unter den Lohn- oder Einkommensteuerpflichtigen die „obersten“ ein Prozent - mit einem Anteil an allen steuerpflichtigen Einkünften von 13,1\% - von der Summe festgesetzter Lohn- und Einkommensteuern $25 \%$; dagegen entrichten die „unteren“ $50 \%$ aller Steuerpflichtigen, immerhin fast 20 Mio. an der Zahl, mit einem Einkünfteanteil von zusammen 14,3\% einen Anteil an allen festgesetzten Lohn- und Einkommensteuern von „nur“ 3,6\%. Der damit vermittelte Eindruck ist aber ebenso banal wie irreführend.

Banal ist diese empirisch unterschiedliche Verteilung zunächst einmal, weil die Lohn- und Einkommensteuer die einzige Steuerart in Deutschland mit einem eingebauten Progressionseffekt ist oberhalb eines Grundfreibetrages zur Steuerverschonung des Existenzminimums der Erwerbstätigen. Insofern spiegelt eine relativ geringe Gesamtsteuerlast der unteren Einkommensgruppe nur die generelle Intention des Systems. Problematisch ist jedoch, dass offenbar die Gruppe mit unteren Einkommen so groß ist (oder anders formuliert: so viele Steuerpflichtige in Deutschland so wenig verdienen), dass sie kaum Steuern zahlen. Tatsächlich lässt sich die untere Hälfte aller Steuerpflichtigen einteilen in fünf Dezile, deren obere Dezilgrenze - gleichzusetzen mit den maximalen Einkünften jeder Dezilgruppe - bei den Jahreseinkünften wie folgt gestaffelt ist (vgl. Statistisches Bundesamt 2011g): erstes bzw. unterstes Dezil: 1.830 €, zweites Dezil: $5.692 €$, drittes Dezil: $10.947 €$, viertes Dezil: $16.609 €$, und schließlich fünftes Dezil: $22.507 €$. Der Begriff der steuerlichen „Einkünfte“ bezieht sich dabei in der Steuerstatistik auf effektive Einkommen nach Abzug von Grundfreibetrag, Werbungskosten und Betriebsausgaben, aber vor Abzug z.B. von Sonderausgaben und erst recht vor Steuern. Die meisten der in der unteren Einkünftehälfte angesiedelten Steuerpflichtigen dürften allerdings aufgrund eines Arbeitnehmerstatus keine Betriebsausgaben und eher wenig Werbungskosten haben. Außerdem ist zusätzlich zu beachten: Der Begriff der Steuerpflichtigen schließt auch zusammen veranlagte Ehegatten ein, die beide erwerbstätig sein können. Mit anderen Worten: Viele der ohnehin schon niedrigen Einkommen in diesem Einkünftebereich werden faktisch von zwei Personen aufgebracht. Die entscheidende Frage beim Blick auf diese untere Gruppe müsste also lauten: Verdienen diese Personen eigentlich genug?

Der zuletzt genannte Aspekt - zusammen veranlagte Ehepartner unter den Steuerpflichtigen - spielt natürlich auch bei den „oberen“ $1 \%$ der Steuerpflichtigen eine Rolle, deren untere Einkünftegrenze bei 175.000 Euro im Jahr 2007 liegt. Ihre Einkünfte können ebenfalls von zwei erwerbstätigen Personen aufgebracht worden sein. Allerdings werden in diesen Einkünfte- bzw. Einkommensregionen Betriebsausgaben und Werbungskosten eine wesentlich größere Rolle spielen. Trotzdem ist hier eher zu fragen: Zahlen diese Kreise eigentlich genug Steuern? Die Frage gilt genauso gut für diejenigen Steuerpflichtigen unterhalb der oberen $1 \%$, z.B. für die oberen $10 \%$ bzw. das oberste Dezil, deren Einkünfte im Jahr 2007 bei $63.478 €$ beginnen. Denn laut Steuerstatistik 2007 beträgt bezogen auf ihre steuerlichen Einkünfte die durchschnittliche Steuerlast der oberen $1 \%$ (10\%) der Steuerpflichtigen $33 \%$ (26,2\%), bezogen auf ihre verfügbaren Einkommen also noch weniger. Bekanntlich haben z.B. gerade Selbstständige und Unternehmer einen gewissen Spielraum, Steuerpflichten zu vermindern, indem sie etwa private Ausgaben als Betriebsausgaben deklarieren. Oder die Steuerpflichtigen geben einen Teil ihrer Einkommen gar nicht an und hinterziehen somit Steuern. Das weiß der Fiskus selbst seit Langem und versucht es mehr schlecht als recht z.B. durch den Ankauf von „Steuer-CDs“ mit den Daten über Steuerflüchtlinge oder durch das deutsch-schweizerische Steuerabkommen einzudämmen. Schließlich kommt auch eine eingehende Untersuchung der Steuerbelastung sehr hoher Einkommen zu dem Ergebnis, dass diese nach den vielen Entlastungen im Durchschnitt zuletzt 
ken zurück. Am Beispiel der Griechenland- und Euro-, Rettungsmaßnahmen“ wird das besonders deutlich. Man liefert sich nach wie vor der „unsichtbaren Weisheit" der ratenden Finanzmärkte aus und verordnet Griechenland ein radikales Sparprogramm, das sozial, gesellschaftlich und ökonomisch kontraproduktiv ist. Der durchs Sparen eingetretene Einbruch des griechischen Wachstums hat das schon hinlänglich gezeigt - und gleichzeitig die Finanznöte Griechenlands nur erhöht. Selbst der Internationale Währungsfonds (IWF) bescheinigt inzwischen auf Basis früherer Erfahrungen solcher Austeritätspolitik negative Wirkungen (Guarjado et al. 2011). Trotzdem wird die Spardosis für Griechenland weiter verstärkt - und wird als Giftdosis beim Patienten wirken (vgl. z.B. Fricke 2011; IMK 2011a). Und die Umsetzung der Sparpolitik scheint unter den Griechen wieder nicht die eigentlichen Adressaten zu treffen, die trotz privaten und betrieblichen Reichtums bisher ihre Steuern weitgehend verweigerten. Schließlich gefährdet die Sparpolitik dort auch deshalb das Vertrauen ins politische System und das „europäische Projekt“. Am Problematischsten aber ist, dass Griechenland nur als vordergründiges Beispiel steht für ähnliche Prozesse in Portugal, Spanien, Rumänien und anderen EU-Ländern. Auch in Deutschland ist angesichts der Kostenrisiken von Banken- und Griechenlandrettung eine Verschärfung der durch die Schuldenbremse ohnehin angelegten Sparpolitik nicht unwahrscheinlich - erst recht, wenn zur selbst verursachten Bremsung der deutschen Exporte aus den europäischen „Krisenländern“ eine fremdbestimmte aus den noch boomenden asiatischen Länder dazukommt und so der einzige und gleichzeitig besonders verwundbare Wachstumsfaktor Export wieder erlahmt.

Bevor also das europäische Wohlfahrts- und Sozialstaatssystem noch mehr infrage gestellt wird als bislang, muss die Politik ihre Lernunwilligkeit endlich aufgeben und einen entschieden neuen Kurs einschlagen. Die deutsche Opposition scheint sich dazu anzuschicken: Alle dortigen Parteien haben Steuererhöhungsvor- schläge entwickelt, die von erhöhten Spitzensteuersätzen bei der Einkommensteuer über eine wieder eingeführte - befristete oder unbefristete - Vermögensteuer bzw. Vermögensabgabe bis hin zu einer Finanzmarkttransaktionssteuer reichen. Doch auch solche Vorhaben, selbst wenn sie nicht auf dem Papier bleiben würden, genügen allein für eine wirkliche Umkehr nicht. Dazu sind neben Finanzierungsmechanismen auch viele Regulierungsmechanismen des Staates völlig neu auszurichten, die als Rahmenbedingungen die negativen Folgen der vorhandenen Verteilung einfangen und die Voraussetzung für eine bessere Verteilung schaffen. Und schließlich ist für eine entschiedenere Umkehr auch eine neue Gesellschaftspolitik notwendig, für die im vorliegenden Heft der WSI-Mitteilungen auch andere Beiträge plädieren.

Hier sollen zur Unterlegung einer solchen Umkehrstrategie nur wenige verteilungspolitische Maßnahmen genannt werden, die die in früheren WSI-Verteilungsberichten geforderten ergänzen:

(1) Spätestens die hessische „Steuerfahnder-Affäre“ aus der Ära des früheren Ministerpräsidenten Koch belegt, dass der deutsche Steuervollzug von der Länder- in die Bundesverantwortung überführt werden muss, um „kleinstaatlichen“ heimlichen Steuersenkungswettbewerb zu unterbinden.

(2) Die erst vor Kurzem eingeführte Abgeltungssteuer auf Zins- und Veräußerungserträge ist in eine Abschlagsteuer umzuwandeln, aber als Quellensteuer mit einem deutlich höheren Steuersatz zu erhalten; dann würde sie - übrigens nach Schweizer Vorbild für schweizerische Steuerbürger nicht nur wie bisher von allen deutschen Kapitalsammelstellen an den Fiskus bei jedem Euroertrag automatisch abgeführt; sie würde zusätzlich wegen ihrer Höhe von z.B. $40 \%$ die betroffenen Steuerpflichtigen zwingen, durch Veranlagung bzw. durch Offenlegung aller ihrer Einkommensverhältnisse gegenüber dem Fiskus wenigstens einen Teil der hohen Abschlagsteuer zurückzuholen.
(3) Das von der jetzigen Bundesregierung ausgehandelte Steuerabkommen mit der Schweiz zur Abgeltung von dort hinterzogenen Steuern durch die Geldanlage deutscher Steuerpflichtiger sollte auf keinen Fall Gesetz werden, weil es immer noch viele Schlupflöcher bietet und vor allem als faktische Steueramnestie ein problematisches politisches Signal setzt (vgl. auch Tax-Justice-Network 2011).

Aus diesen kleinteiligen Vorschlägen darf allerdings nicht der Schluss gezogen werden, die Verteilungsfragen seien nachrangig angesichts der anstehenden Herausforderungen, wie zuallererst die Bewältigung der „Europäischen Krise“. Im Gegenteil: Weil die aktuelle Krise des Euro, des Projekts Europa, der politischen Glaubwürdigkeit ursächlich mit Ungleichheiten $\mathrm{zu}$ tun hat, die früher schon nationale Krisen in Form von sozialer Ungerechtigkeit, gesellschaftlicher Spaltung und unzureichender Binnennachfrage erzeugt haben, folgen daraus möglicherweise im Zusammenwirken sogar Beschädigungen des demokratischen Systems. Der soziale Unfrieden in Griechenland angesichts der aufgezwungenen Sparpolitik ist vielleicht nur ein Vorspiel ${ }^{6}$. Aber man sollte es auf den Eintritt des Endspiels gar nicht erst ankommen lassen und die Verteilungsfrage rechtzeitig als prioritäre Schlüsselfrage angehen und die Probleme lösen. Der politische Ernst, der dabei zum Zuge kommen müsste, geht aus der Formulierung eines Journalisten treffend hervor, indem er das Motto der amerikanischen Unabhängigkeitsbewegung gegen die Steuer pressende englische Kolonialmacht umdreht: „No representation without taxation" (Schieritz 2011) - und gemeint ist natürlich: Keine wirkliche Demokratie ohne gerechte und ergiebige Besteuerung; oder noch kürzer: ohne Umverteilung von oben nach unten.

6 Zu alternativen Lösungswegen des griechischen "Problems", das auch von Griechenland selbst verschuldete Reformdefizite einschließt, siehe z.B. IMK 2011b. 
Bach, S./Corneo, G./Steiner, V. (2011): Effective Taxation of Top Incomes in Germany, Studie im Auftrag des Bundesministeriums für Finanzen, Berlin, (http://www.wiwiss.fu-berlin.de/institute/finanzen/ corneo/dp/BCS_EffectTaxRate_2011-06-20.pdf)

Brenke, K. (2011a): Anhaltender Strukturwandel zur Teilzeitbeschäftigung, in: DIW Wochenbericht 78 (42), S. 3-12

Brenke, K. (2011b): Teilzeitarbeit: Immer mehr arbeiten immer weniger, in: DIW Wochenbericht 78 (42), S. 13

Brenke, K./Wagner, G. (2011): "Nötig sind kräftige Lohnerhöhungen“. DIW nennt neue Daten zur Einkommensentwicklung, in: Frankfurter Rundschau vom 2.8.2011, S. 15

Bundesministerium der Finanzen (BMF) (2011): Ergebnis der 138. Sitzung des Arbeitskreises "Steuerschätzungen“ vom 10.-12. Mai 2011 in Fulda, http://www.bundesfinanzministerium.de/nn_4156/DE/ Wirtschaft__und__Verwaltung/Steuern/Steuerschaetzung__ einnahmen/Ergebnis__der__Steuerschaetzung/1105131a6002,template Id = raw, property=publicationFile.pdf

Bundesregierung (2011): Armutssituation in der Bundesrepublik Deutschland. Antwort auf die Anfrage der Linksfraktion, BundestagsDrucksache 17/6043, Berlin

Corneo, G. (2011): „Die Reichen kommen zu leicht davon“, Interview mit Zeit Online vom 06.07.,http://www.zeit.de/wirtschaft/2011-07/ interview-corneo-reichensteuer

Der Tagesspiegel (2011): Gehaltsunterschiede in Ost und West - „Die Menschen kommen nicht zurück“" Ausgabe vom 02.10., Berlin http:// www.tagesspiegel.de/wirtschaft/die-menschen-kommen-nichtzurueck/4679254.html

Deutsche Bundesbank (2010): Ertragslage und Finanzierungsverhältnisse deutscher Unternehmen im Jahr 2009, in: Monatsbericht Dezember, S. $31-45$

Deutsche Bundesbank (2011): Ergebnisse der Gesamtwirtschaftlichen Finanzierungsrechnung für Deutschland 2005 bis 2010, Statistische Sonderveröffentlichung 4/2011, nur online: www.bundesbank.de Fitoussi, J.-P./Stiglitz, J. (2009): The way out of the crisis and the building of a more cohesive world, in: Observatoire Français des Conjonctures Economiques (OFCE), document de travail No.2009-17 Fricke, T. (2011): „Huch, der Patient ist tot“, in: Financial Times Deutschland vom 14.10., http://www.ftd.de/politik/europa/: kolumne-thomas-fricke-huch-der-patient-ist-tot/60115883.html Grabka, M. (2011): Entwicklung des realen Nettoerwerbseinkommens von abhängig Beschäftigten auf Basis von Monatseinkommen, Berlin (unveröffentlichte Tabellen)

Guajardo, J./Leigh, D./Pescatori, A. (2011): Expansionary Austerity: New International Evidence, IMF Working Paper 11/158, New York

Horn, G. (2011): Des Reichtums fette Beute. Wie die Ungleichheit unser Land ruiniert, Frankfurt/New York

Institut für Makroökonomie und Konjunkturforschung (IMK) (2011a): Voraussetzungen einer erfolgreichen Konsolidierung Griechenlands, IMK-Report (66), Düsseldorf

Institut für Makroökonomie und Konjunkturforschung (IMK) (2011b): Nothing learned from the crises? Some remarks on the Stability Programmes 2011-2014 of the Euro area governments, IMK-working paper (11), Düsseldorf

Keller, B./Seifert, H. (2011): Atypische Beschäftigung und soziale Risiken: Entwicklung, Strukturen, Regulierung, Friedrich-Ebert-Stiftung, WISO-Diskurs, Bonn
Manager-Magazin (2011): Nachgezählt: Mehr als 800 Superreiche in Deutschland, Ausgabe vom 01.06., http://www.manager-magazin.de Merrill Lynch/Capgemini (2011): World Wealth Report 2011, http:// www.de.capgemini.com/insights/publikationen/world-wealthreport-2011/)

Möller, J. (2011): Qualifikationsbedingte Lohnunterschiede. Wer kriegt wie viel Butter aufs Brot?, in: IAB-Forum (1), S. 4-13

Moore, C. (2011): I'm starting to think, that the Left might actually be right, in: The Telegraph vom 22.07.2011, http://www.telegraph.co.uk/ news/politics/8655106/Im-starting-to-think-that-the-Left-mightactually-be-right.html

Rodrik, D. (2011): Das Globalisierungs-Paradox - Die Demokratie und die Zukunft der Weltwirtschaft, München

Schäfer, C. (2010): Zukunftsgefährdung statt Krisenlehren - WSI-Verteilungsbericht 2010, in: WSI-Mitteilungen 63 (12), S. 636-645 Schieritz, M. (2011): Raus aus dem Schuldenloch, in: Die Zeit Nr. 31. vom 28.07., http://www.zeit.de/2011/31/Euro-Rettung-Steuern Schirrmacher, F. (2011): Ich beginne zu glauben, dass die Linke recht hat, in: Frankfurter allgemeine Sonntagszeitung vom 14.08., http:// www.faz.net/aktuell/feuilleton/buergerliche-werte-ich-beginne-zuglauben-dass-die-linke-recht-hat-11106162.html

Statistisches Bundesamt (2011a): Revision der Volkswirtschaftlichen Gesamtrechnungen 2011 für den Zeitraum 1991 bis 2010, in: Wirtschaft und Statistik, September, S. 825-865

Statistisches Bundesamt (2011b): Volkswirtschaftliche Gesamtrechnungen - 2. Vierteljahr 2011, Fachserie 18, Reihe 1.2, Wiesbaden Statistisches Bundesamt (2011c): Bruttoinlandsprodukt in der ersten Jahreshälfte 2011 - Vorläufige Ergebnisse, in: Wirtschaft und Statistik (9), S. 866-898

Statistisches Bundesamt (2011d): Beschäftigungszuwachs 2010 zu großen Teilen von Zeitarbeit getragen, Pressemitteilung Nr. 217 vom 19.07., Wiesbaden

Statistisches Bundesamt (2011e): Verdienste und Arbeitskosten - Arbeitnehmerverdienste 2010, Fachserie 16 Reihe 2.3, Wiesbaden Statistisches Bundesamt (2011f): Hoher Anstieg der Bruttoverdienste setzt sich im 2. Quartal 2011 fort, Pressemitteilung Nr. 347 vom 21.9., Wiesbaden

Statistisches Bundesamt (2011g): 1 \% der Steuerpflichtigen zahlt $25 \%$ der Einkommensteuer, Pressemitteilung 378 vom 12.10., Wiesbaden Statistisches Bundesamt (2011h): Erste Ergebnisse der Lohn- und Einkommensteuerstatistik 2007 - Ergänzungsmaterial zur Pressemitteilung 378 vom 12.10., Wiesbaden

Statistisches Bundesamt/Wissenschaftszentrum Berlin für Sozialforschung (WZB)/Bundeszentrale für Politische Bildung (BpB) (2011): Datenreport 2011. Der Sozialbericht für Deutschland, Bonn Tax-Justice-Network (2011): Für eine Handvoll Euro und Daten Deutschland kapituliert vor dem Schweizer Bankgeheimnis, Version vom 22.09., http: //www.taxjustice.net

van Treeck, T. (2011): Soziale Ungleichheit führt zu wirtschaftlicher Instabilität, in: Gegenblende - Online-Magazin des DGB, September 2011, http://www.gegenblende.de

Wilkinson, R./Pickett, K. (2010): Gleichheit ist Glück: Warum gerechte Gesellschaften für alle besser sind, Berlin 